\title{
Cellular and molecular biology of the Beta cell
}

\author{
Work group chairman: D. F. Steiner ${ }^{1}$ \\ Report prepared by: D. E. James ${ }^{2}$ \\ ${ }^{1}$ Howard Hughes Medical Institute, Chicago, Illinois, and ${ }^{2}$ Department of Cell Biology and Physiology, Washington University Medical
School, St. Louis, Missouri, USA
}

Summary. Considerable progress has been made in our understanding of islet-cell function and its relationship to regulation of whole body glucose metabolism. At the genetic level, the regulatory regions in islet-specific genes are being characterised. Transcription factors that interact with these regions have been cloned and these will be instructive in elucidating how islet-specific genes are regulated during development and regeneration. Identification of the enzymes responsible for proteolytic conversion of proinsulin to insulin represents a major advance in understanding prohormone processing. Cleavage of proinsulin is mediated by at least two prohormone convertases (PC3/PC1 and PC2). Their activity is regulated by an acidic gradient between the Golgi and secretory granules and by calcium ions. It is not yet clear how insulin or the PC's are specifically diverted into the regulated secretory pathway. Regulation at this step may be defective in some diabetic patients resulting in relatively elevated circulating proinsulin levels.

Specific features of GLUT 2 and glucokinase (GK), proteins that regulate Beta-cell glucose transport and phosphorylation, indicate that these may be key components of the glucose sensor. GLUT 2 is necessary to reconstitute glucose-sensitive insulin secretion in pituitary tumour cells expressing a proinsulin cDNA. Furthermore, the expression of GLUT 2 in Beta cells, but not in hepatocytes, is decreased in diabetes mellitus. However, under normal circumstances GK is probably rate limiting for Beta-cell glucose utilisation. Thus, it is likely that both GLUT 2 and GK determine the set point for glucosestimulated insulin secretion.

Elucidation of distal effectors that regulate insulin secretion is also crucial to our understanding of Beta-cell function. Elevations in cytosolic $\mathrm{Ca}^{2+}$ are essential for stimulus secretion coupling. A novel second messenger, cyclic ADP ribose, has been implicated as a regulator of glucose-stimulated $\mathrm{Ca}^{2+}$ release from the Beta-cell endoplasmic reticulum. These and other recent advances provide optimism for the ultimate development of an artificial Beta cell capable of making insulin and releasing it in response to glucose.

Key words: Insulin gene, expression, precursor processing, glucokinase, glucose transport, signal transduction.

\section{Introduction}

Recent studies on the cellular and molecular biology of the Beta cell have highlighted several novel metabolic attributes that underlie its complex physiological role in regulating glucose homeostasis. Identification and functional analysis of several new Beta-cell specific genes have led to revisions of our view of the phenotype of this cell, and have provided a framework for future investigations of its functional integration in the organism as a whole. It is increasingly clear that a large number of gene products, aside from insulin, are required to maintain the normal differentiated state of the Beta cell. These include specialised proteins that regulate the various functions of Beta cells, among them glucose sensing and stimulus-secretion coupling, insulin biosynthesis, transport and processing, and also a growing number of transcriptional regulatory factors that serve to differentiate and maintain this unique phenotype. As is often the case in biological research, these advances have answered some questions while raising many others. The participants of this work group discussed recent progress in this area and attempted to define some of the important goals that should be attainable in the future.

\section{State of the art}

Islet-specific gene expression. Several important regulatory elements and factors have been identified that control the transcription of three islet-specific genes (insulin, glucagon 


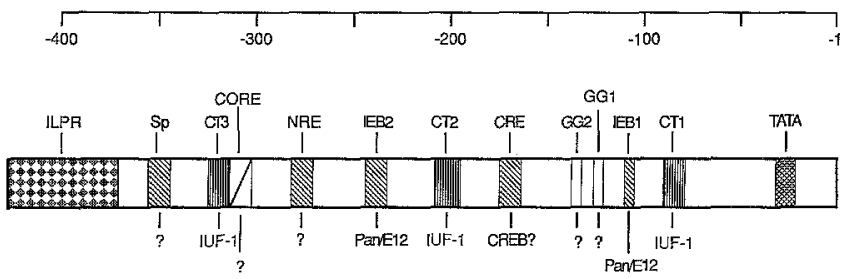

Fig. 1. Proposed regulatory sequences of the human insulin gene upstream promoter. The name for each motif is given above and the corresponding DNA binding protein is identified below the schematic [2]

and glucokinase). Discussion focussed on upstream regulatory elements in these genes that appear to bind either Beta-cell- or islet-cell-specific factors (Fig. 1). The most important and highly conserved site in the insulin gene is the insulin enhancer box (IEB-1) that binds a helixloop-helix factor (HLH) [1]. However, other elements and factors appear to be involved. A new Beta-cell-specific factor has been identified [1]. This factor, termed insulin promoter factor-1 (IPF-1), contains a homeodomain. Experiments have shown that this factor binds to and transactivates an insulin upstream promoter element (TAATGGG) that occurs at a single site in the rat insulin I gene. Related or identical sequences occur at three sites in the human insulin promoter-enhancer region (the so-called "CT boxes 1-3"). Studies by Docherty et al. [2] have also demonstrated the existence of a Beta-cell-specific factor, which they have called IUF-1, that binds to the CT elements. This element also appears at three sites in the glucokinase gene promoter (Shelton KD, Khoor MA, Magnuson $M$, unpublished data) thus raising the possibility of some coordinate regulation of the insulin and glucokinase genes, although other aspects of the expression of these genes are distinct.

A powerful negative regulatory element (NRE) is also present upstream of the human insulin gene [2]. This NRE acts as a silencer, i.e., functions independently of position and orientation, and is to some degree promoterindependent. The NRE functions in both Beta cells as well as in other cells, and thus is not responsible for repression of insulin gene expression in non-Beta cells. An adjacent positive element modulates the activity of the NRE.

Recent results with Isl-1, an LIM-homeodomain protein expressed only in the endocrine portion of the pancreas, suggests a role for this factor in islet cell development more generally [3]. The concept of Isl-1 as a developmental determinant of both primitive brain (autonomic functions) and of a variety of neuroendocrine cells was supported by studies characterising both the temporal and spatial pattern of its expression in developing chick and mouse embryos.

Relatively less information is available on the regulation of the glucagon gene, which is expressed in islets, intestine and adult brain. Drucker reported his recent studies on the expression of the glucagon promoter in transgenic mice. Using a larger promoter region than previously described $(-2 \mathrm{~kb}$ of the rat 5 ' flanking DNA), transgene expression was identified in intestine in addition to the alpha cells of the islet and brain [4]. Future studies will be directed at elucidating the regulatory elements and factors that direct intestinal cell-specific expression of the glucagon gene.

It is likely that a variety of additional transcription factors to those described above are involved in specifying the phenotype of the pancreatic Beta cell. Magnuson reported the cloning of a factor in his laboratory termed Beta-cell-enriched zinc finger protein (BZP) from insulinoma cells. This large factor $(\sim 114 \mathrm{kDa})$ contains eight zinc finger motifs of the Kruppel class. Studies directed at elucidating the sites of its expression and its function were reported to be in progress (Franklin A, Shelton K, Magnuson M, unpublished data).

Islet differentiation. During pancreas ontogeny the four classical and highly distinct islet-cell types derive from a common stem cell by passing through a transient phase characterised by co-expression of several hormone genes (initially glucagon and insulin) within the same cell [5]. This can be viewed as a two-step process characterised by general hormone gene activation leading to mixed phenotypes, followed by selective inactivation of inappropriate genes to yield the final mature islet cell phenotypes. The "mixed phenotype" feature is also reflected in tumour biology of the endocrine pancreas where most if not all tumours are heterogeneous, expressing multiple phenotypes. Madsen has proposed that the tight coupling between islet stem-cell proliferation and activation/inactivation of hormone genes may be reconstituted when mature islets are induced to proliferate following transformation and addition of growth factors. Madsen and colleagues have devised approaches to generate clonal rat islet tumour lines (MSL cells) that are highly heterogeneous, thus displaying characteristics of the stem-cell phenotype [6]. Many neuroendocrine proteins (e.g. Type 1 diabetes autoantigens, GAD64 and GAD67), polypeptide hormones and neurotransmitters (e.g. GABA, neurotensin) are expressed in MSL cells. Passage of these cells in vivo results in dramatic shifts in phenotype which may prove useful for identifying extrinsic factors that regulate the differentiated state in vivo. This also appears to provide a useful system for identifying new transcription factors or for further characterising transcription factors (such as those described above) that regulate cell-specific islet gene expression [7].

Insulin synthesis, processing and secretion. Insulin processing in islet Beta cells has been extensively studied by Orci and colleagues [8]. Proinsulin is co-translationally inserted into the lumen of the endoplasmic reticulum (ER) via a cleavable signal sequence at its amino terminus. In the ER, proinsulin assumes its appropriate tertiary structure via folding and the formation of disulphide bonds. The native protein transits through the lumen of the ER and the Golgi cisternae. At the trans face of the Golgi, in a 
compartment known as the trans Golgi reticulum (TGR), proinsulin is specifically packaged into clathrin coated vesicles that bud from the TGR. This is a major point of regulation because several different classes of vesicles may exit from the TGR, distinguished primarily on the basis of their specific cargo and in some cases their eventual destinations. Two distinct routes for secretory proteins have been described, namely the regulated and constitutive pathways. The factors that determine pathway specificity for secretory proteins have not been ascertained. Proinsulin is efficiently targeted into the regulated pathway via clathrin coated vesicles which mature into secretory granules. In contrast to constitutive secretory vesicles, insulin secretory granules are retained in the cytoplasm of the Beta cells until their exocytic movement is triggered by an elevation in blood glucose. Processing of proinsulin into insulin and C-peptide has been shown to occur in the secretory granules as they mature $[8,9]$.

A broad description of many of the individual steps that intervene between insulin translation and its actual secretion from the Beta cell have been provided by morphological and biochemical experiments. However, many fundamental questions remain to be answered. How is proinsulin specifically targeted to the secretory granules? What factors regulate cleavage of proinsulin into insulin and the connecting peptide (C-peptide) and exactly where in the cell is this process initiated? Most importantly, what is the mechanism by which glucose and other secretagogues, such as arginine, stimulate the exocytosis of the insulin storage granules? Despite a large body of work, little is known regarding the factors that regulate the sensitivity of Beta cells to glucose and whether specific biochemical defects may underlie defects in insulin production and secretion in the diabetic state. Because our understanding of the regulation of insulin processing and secretion is still only rudimentary, it is not yet possible to precisely define points of regulation that may be disturbed in the disease state.

Insulin sorting. Halban and co-workers [9] have undertaken a series of studies aimed at understanding how proinsulin is directed to secretory granules. There are two hypotheses to account for the targeting of soluble proteins such as proinsulin to the regulated secretory pathway in endocrine cells. It has been proposed that a unique sequence motif or structural domain of prohormones or hormones may bind to specific receptor molecules in the TGR that direct them into secretory granules. However, the lack of obvious homology in primary amino acid sequence between various secreted proteins and the high local concentrations of hormone in the TGR that are generated prior to budding of coated granules argue against this. The paradigm for this type of sorting is that of retention of soluble proteins in the ER. All soluble ERretained proteins share a common sequence motif (KDEL), and there is no evidence for a concentration of these proteins throughout different regions of the ER [10].
A stronger case can therefore be made for the second hypothesis which suggests that targeting to the regulated pathway is mediated via the formation of proinsulin oligomers or aggregates in the TGR. By constructing deletion and point mutations in the proinsulin molecule, a number of investigators have attempted to identify putative targeting domains. To date, however, no sequence motifs that result in targeting of soluble proteins to the regulated pathway have been identified. Although a point mutation at residue B10 (His->Asp) in proinsulin redirects some of the mutant proinsulin to the constitutive pathway in AtT20 cells [9], as described by Steiner, this mutation may indirectly alter proinsulin targeting due to the enhanced binding affinity of the B10 mutant to the insulin receptor. Short deletions from the $\mathrm{N}$-terminal region of the C-peptide have been performed by Halban's group. These mutations, which do not affect targeting of proinsulin to granules, appear to impair insulin processing. A major limitation of this type of approach is that point mutations or deletions may alter targeting simply by disrupting the native structure of the protein. To further explore the basis for the specificity of proinsulin targeting and processing, Halban's group has expressed proinsulin in cells that normally do not exhibit regulated secretion. When expressed in an hepatocyte cell line (Fao), proinsulin appeared to undergo at least partial conversion to insulin. Interpretation of these data will require elucidation of endoprotease expression and activity in these cells. It is likely that a more definitive approach toward understanding insulin processing will require genetic strategies similar to those often used in yeast.

Steiner's group has also performed experiments using the HisB10Asp proinsulin mutant [9]. In agreement with the studies in AtT20 cells, this mutant human gene, which was targeted in its expression to the Beta cell using transgenic mice, appeared to be missorted into the constitutive pathway. Of note, the B10Asp mutant proinsulin had a marked increase (four- to five-fold) in its affinity for binding to the insulin receptor. Based on this finding, it has been proposed by Steiner and co-workers that insulin receptors may constantly recycle between the TGR and the cell surface via the constitutive pathway, thereby binding some of this mutant proinsulin in the TGR and diverting it away from the regulated pathway. However, further basic cell biological studies of insulin sorting will be required to confirm this provocative hypothesis, i.e. that the secretory granule pathway may be a default pathway in neuroendocrine cells like the Beta cell.

If indeed proinsulin condensation is implicated in its sorting to secretory granules, major questions that need to be addressed in the future include the biophysical nature of such proinsulin aggregates and how they might be formed. A more fundamental question is how the processing enzymes can efficiently catalyse the conversion of proinsulin to insulin if it exists as an amorphous condensed structure in newly synthesised granules. Once these and other questions are answered, we can begin to examine the 
effects of glucose and other factors on these steps in the insulin biosynthetic pathway.

Proinsulin converting enzymes. An area which has undergone major advances in recent years is that of the enzymology of conversion of proinsulin to insulin. Studies using secretory granule extracts indicate that two calciumdependent enzymes are involved in the initial cleavage of the prohormone. Type 1 enzyme recognises the Arg-Arg sequence at the B chain/C-peptide junction whereas a type 2 enzyme recognises predominantly the Lys-Arg sequence at the C-peptide A chain junction in human proinsulin. It has been proposed by Hutton and co-workers that this sequence specificity, along with differences in sensitivity to calcium and $\mathrm{pH}$, may provide a mechanism whereby prohormone conversion is restricted to the secretory granule and may be regulated differentially in various tissues [11]. However, until recently the enzymes responsible for these cleavages remained elusive. The molecular identification of a family of endoproteases that appear to be responsible for this catalytic conversion has afforded rapid progress.

Using yeast genetics, it was shown a number of years ago that an endoprotease known as Kex2 was the major protease responsible for maturation of the constitutively secreted pheromone $\alpha$-mating factor. The identification of Kex 2 mammalian counterparts quickly led to the elucidation of a new gene family of mammalian endoproteases by Steiner's group and others [12]. Four members of this new endoprotease family have been identified in mammals: Furin/Pace, a Golgi associated membrane protein which is the mammalian homologue of Kex2; PC2 and PC1/PC3, non-membrane bound, calcium

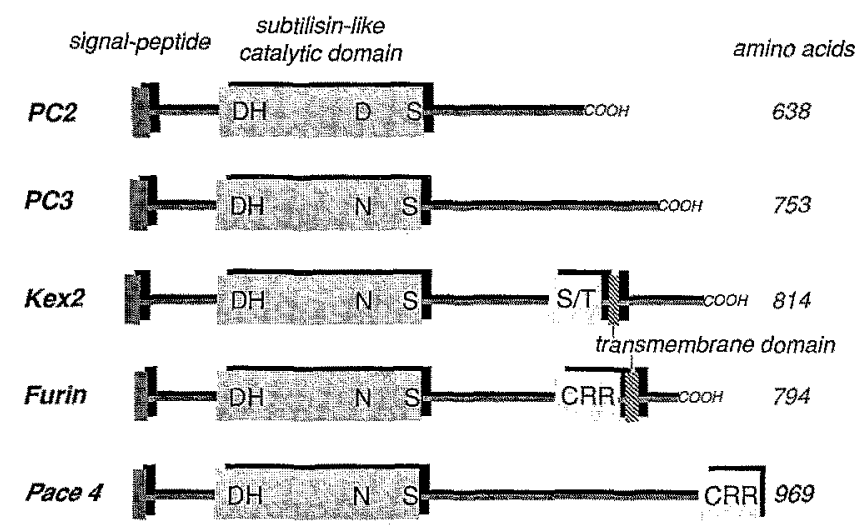

Fig. 2. The subtilisin-related proprotein convertase (SPC) family. Four mammalian homologues of the yeast proprotein convertase, $\mathrm{Kex} 2$, are depicted diagrammatically above. Each protease contains a subtilisin-like serine protease domain with essential catalytic residues arranged as shown in the large boxed region ( $D=$ aspartic acid, $H=$ histidine, $N=$ asparagine, $S=$ serine). Other structural features include $S / T$ serine/threonine rich region and CRR-cysteine rich region. Structural similarity extends throughout approx. the first 600 amino acids of all five proteases shown but is highest in the catalytic domains. These enzymes are described in greater detail in [12] and in the text (Data on Pace 4 is from Kiefer et al. DNA and Cell Biol 10:757, 1991.) activated secretory granule processing enzymes; and Pace 4, a furin-like enzyme, also expressed at high levels in liver (as is furin) and possibly involved in proalbumin processing (Fig. 2). Each of these proteases has a similar subtilisin-like catalytic domain. Furin (and perhaps Pace 4) require a somewhat different cleavage recognition signal, consisting minimally of Arg-X-X-Arg, which appears in many viral glycoprotein precursors as well as in many growth factors, receptor precursors (insulin, IGF-1) and coagulation zymogen precursors (factors IX, XI, etc). With the use of peptide-specific antibodies and specific DNA probes for each of these molecules it has been possible to learn a great deal about the biochemistry of insulin processing. Two of the above proteases (PC2 and PC3) show a similar tissue distribution to the polypeptide hormones and neurotransmitters and are strong candidates for the prohormone convertases. PC2 is the major isoform in Beta cells and is expressed only in neuronal and neuroendocrine cells. By means of immunoelectron microscopy, $\mathrm{PC} 2$ has been localised to the secretory granules in islet Beta cells (Smeekens SP, Swift H, Steiner DF, unpublished data). Similar studies on the subcellular localisation of furin and PC3 in islets need to be performed. One interesting structural feature pointed out by Steiner is that $\mathrm{PC} 2$ has an Asp residue in the catalytic domain which is conserved across species. This residue is invariably Asn in PC3, furin, Kex2 and the subtilisins and thus may play an important role in regulating the activity of PC2 since Asp will be able to function only when its side chain carboxylic group is protonated at acidic $\mathrm{pH}$. All of these findings taken together suggest that the SPC enzymes play a general role in processing of soluble prosecretory proteins in the body.

Using a pulse chase analysis, Hutton's group has found that in islets PC2 is glycated and that it undergoes dibasic cleavage somewhere in the vicinity of the TGR resulting in the formation of a $65 \mathrm{kDa}$ mature product. The $65 \mathrm{kDa}$ form, but not the immature forms, is secreted into the medium in response to glucose stimulation of insulinoma cells. This is consistent with the notion that PC2 is a soluble protein that is localised to the insulin secretory granules. The processing and activation of $\mathrm{PC} 2$ may also be an important locus for regulation because defects here may result in improper processing of proinsulin. Indeed, using an antisense approach to knock out PC2, Steiner's group has shown that immature insulin products accumulate under these conditions.

With these proteases in hand, it has been possible to study insulin processing in more detail. Hutton's group used biochemical procedures to show that the insulinoma granule type 2 endoprotease activity that they had previously defined is probably mediated via PC2. In addition, Xenopus oocytes microinjected with PC2 mRNA express a proinsulin convertase that is indistinguishable from the type 2 enzyme activity (Shennan $K$, Docherty $K$ and Hutton J, unpublished results). Similar experiments to those described for $\mathrm{PC} 2$ were performed using antibodies 
specific for PC3 and suggest a possible parallel between that gene product and the type 1 enzyme. PC2 and PC3 also process other prohormones, as their neuroendocrine tissue distribution would predict. Several groups have shown that murine pro-opiomelanocortin (POMC), chromogranin $A$ and other precursors are processed in vivo or in vitro by $\mathrm{PC} 3$ and $\mathrm{PC} 2$ to yield their physiologically relevant mature forms [12]. Proalbumin and the insulin $\alpha \beta$ receptor precursor, which are processed in the constitutive pathway in vivo, are both cleaved in vitro by the type 1 or type 2 islet protease activities. However, other evidence suggests that other members of the SPC family of proteases, most likely furin or Pace 4, are responsible for these cleavages in vivo. These studies raise many new questions about insulin processing and Beta-cell function: What is the substrate specificity of these endoproteases in vivo and is their activity subject to any type of regulation? How does diabetes influence the activity or expression of these enzymes? And, finally, how are these enzymes specifically targeted into secretory granules?

Role of amylin (IAPP) - a new secretory product of the Beta cell. The recent and surprising discovery that the Beta cell makes and secretes a peptide related to the neuropeptide calcitonin gene related peptide (CGRP) has provided a new challenge to our concepts of normal Betacell physiology. Moreover, the tendency of the 37 amino acid, islet amyloid polypeptide (IAPP)/amylin to form amyloid fibrils in certain species, including man, has introduced a possible pathogenic role for amylin. First discovered by Westermark (Uppsala) and Cooper (Oxford), the structure of its precursor and of its gene and chromosomal localisation have been elucidated in man and in several other mammalian species [13]. Amino acid differences between IAPP products identified from different species appear to account for the ability of this protein to form fibrils or not. The peptide is expressed almost exclusively in the Beta cells of the adult islets and IAPP is co-secreted with insulin in amounts of approximately $1-2 \%$ the level of insulin. While an insulinresistant-like effect of amylin in muscle has been reported, the cognate receptor has not yet been identified and the precise site of action of IAPP has not been mapped. A controversy that surrounds the role of IAPP in human insulin resistance is as to whether its physiological levels are high enough to affect muscle glucose metabolism. While amyloid deposition occurs in islets of diabetic patients, overproduction of IAPP does not appear to be a feature of human Type 2 diabetes. No defects in the IAPP gene on chromosome 12 have been found in a series of diabetic patients. More work is clearly required to elucidate the role of this protein in insulin resistance and in the pathogenesis of amyloid deposits.

Glucose sensing and Beta-cell function. Considerable progress has been made in the area of stimulus secretion coupling in islets with the identification of several key molecules that regulate glucose entry and metabolism in Beta cells. A family of five facilitated mammalian glucose transporters (GLUT 1-5) have been identified, the most distinguishing feature of which is their unique tissue distribution [14]. The high $\mathrm{K}_{\mathrm{m}}$ isoform known as GLUT 2 is expressed in Beta cells as well as in intestine, kidney and liver. This glucose transporter is not expressed by other pancreatic endocrine cells. The glucose phosphorylating enzyme specific to Beta cells, glucokinase (GK), has also been cloned and, like GLUT 2 , has a high $K_{m}$ for glucose. The $\mathrm{K}_{\mathrm{m}}$ 's for glucose of GLUT 2 (18 mmol//) and GK (8$10 \mathrm{mmol} / \mathrm{l})$ may allow glucose uptake and phosphorylation in Beta cells to increase in proportion to the elevation in blood glucose as it occurs in the postprandial state. In view of these kinetic properties, it has been suggested that both GLUT 2 and GK may play important roles in glucose sensing. Under normal physiological conditions GK rather than GLUT 2 appears to be rate limiting for glucose utilisation in Beta cells [15]. However, this balance may be altered in certain disease states if the expression or activity of GLUT 2 is impaired. In view of these developments a great deal of effort has been devoted to the study of these molecules in islets in both the normal and diabetic state.

In a number of rodent models of both Type 1 and Type 2 diabetes GLUT 2 expression in Beta cells is markedly reduced [16], whereas its expression in liver and kidney remains unchanged. The extent of reduction in GLUT 2 expression is proportional to the severity of diabetes as determined by the glycaemic level. Thus, it has been suggested that reduced GLUT 2 expression may be a major contributor to Beta-cell dysfunction in diabetes. However, if the levels of GK fall in tandem, an important role for this enzyme in the diabetic state may also be implicated (e.g. see Genetic Control of Diabetes Mellitus). Technical constraints have thus far prevented GK measurements in diabetic pancreas. However, this would indeed be an area for future fruitful research, now that DNA probes and antibodies are available.

To assess the factors that control GLUT 2 expression in Beta cells of diabetic animals, Thorens and co-workers have performed transplantation experiments. By transplanting GLUT 2 defective islets from $\mathrm{db} / \mathrm{db}$ mice under the renal capsule of normal mice, it was shown that GLUT 2 expression returned to normal within 2 weeks. Conversely, transplantation of normal islets into either $\mathrm{db} / \mathrm{db}$ mice or streptozotocin diabetic mice resulted in a marked decrease in GLUT 2 expression similar to that observed in $\mathrm{db} / \mathrm{db}$ islets. These results implicate systemic control of GLUT 2 expression in islets. In view of the potential role of impaired GLUT 2 levels in diabetic pancreas it will be important to characterise the factors that regulate GLUT 2 levels in the diabetic state. In addition, since these studies have great relevance for potential transplantation therapy in humans, considerable effort should be invested to identify the putative circulating factor(s) responsible for this effect.

Gut hormones (GIP, GLP1) have long been known to 
potentiate glucose-stimulated insulin secretion. However, little is known concerning the mechanism of this effect. Islets appear to contain a GLP1 receptor which implicates this as a potentially interesting incretin hormone. Using a Cos cell expression library, Thorens' group has cloned the GLP1 receptor from rat islets. This clone encodes a protein that has seven putative transmembrane spanning domains, indicating that it is probably a heterotrimeric G-proteincoupled receptor. However, the GLP1 receptor appears to be a member of a novel subfamily of G-protein-coupled receptors because it shares high primary amino acid sequence homology only with the receptors for calcitonin, secretin and parathyroid hormone. This achievement provides a solid basis for furthering our understanding of gut hormone control of insulin secretion. Moreover, Bell and co-workers [17] have recently identified several somatostatin receptor cDNAs and have shown certain of these to be expressed in the islets of Langerhans, as well as in the central nervous system. This work should add an additional perspective to our rapidly expanding knowledge of the molecular basis for the control of insulin secretion.

Artificial islets. A major goal for diabetes therapy is to genetically engineer an insulin-secreting artificial Beta cell. Newgard's group has moved one step closer to this goal with its studies on the anterior pituitary cell line AtT20ins. These cells are like Beta cells in that they have been stably transfected with proinsulin cDNA and secrete insulin in response to dbcAMP, but unlike Beta cells they do not secrete insulin in response to glucose. Newgard and coworkers have found that stable transfection of these cells with the GLUT 2 cDNA confers glucose-stimulated insulin secretion, increased insulin content and glucose potentiation of several non-glucose secretagogues [18]. The only caveat in these studies is that maximal glucose stimulated insulin secretion occurred at a glucose level of 10-50 $\mu \mathrm{mol} / 1$, well below the physiological range. One potential explanation for this finding, as described by Newgard, is that although AtT20ins cells express GK, the hexokinase:GK ratio is substantially greater in these cells than in islets, where GK activity predominates at physiological glucose concentrations. Studies are currently underway in Newgard's laboratory to manipulate the hexokinase:GK ratio in AtT20ins cells by genetic means in the hope that the $\mathrm{K}_{\mathrm{m}}$ for glucose-stimulated insulin secretion can be elevated into the physiological range.

Beta-cell heterogeneity. If Newgard's hypothesis, that the level of GK is a critical determinant of the glucose sensitivity of insulin secretion in AtT20ins cells, is correct, this implies an important role, as originally suggested by Meglasson and Matschinsky [15], for GK as a glucose sensor in Beta cells. However, little is presently known about the regulation of this enzyme in islets. Magnuson and Jetton [19] have used GK-specific antibodies to immunolocalise GK in the rat pancreatic islet. GK was only expressed in Beta cells but surprisingly there was considerable heterogeneity in GK levels between individual Beta cells (Fig. 3).

This observation was confirmed in transgenic mice expressing fusion genes driven by the upstream GK promoter, suggesting that the Beta-cell heterogeneity in GK expression is regulated at the transcriptional level. These investigations are particularly interesting in view of previous studies [20] showing a similar heterogeneity in glucose-stimulated insulin secretion among individual Beta cells. These studies also provide further support for the proposed role of GK as an important component of the Beta-cell glucose sensor. However, the physiological role of this heterogeneity in Beta-cell expression of GK remains unclear. One possibility is that this may provide the basis for the exquisite sensitivity of the islet to changes in the blood glucose concentration. This model raises the interesting possibility that individual Beta cells may secrete insulin in an "all or none" fashion, similar to the generation of the action potential in neurons, and that the ability of the islet to secrete insulin in proportion to the ambient glucose concentration may reflect a recruitment of individual Beta cells into an active secretory state. Based on the work of Newgard and colleagues, it is likely that the low GKexpressing Beta cells may respond at lower glucose levels whereas the high GK-expressing cells may respond mainly

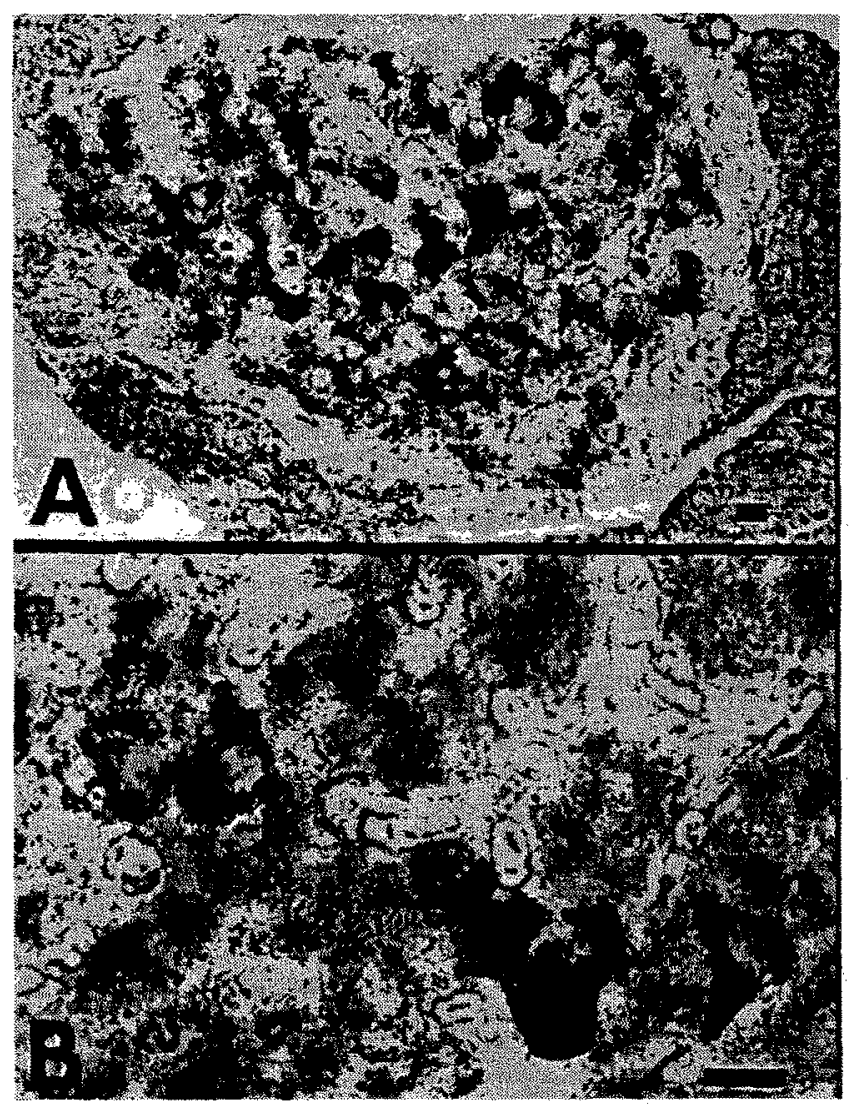

Fig. 3. Illustration of glucokinase heterogeneity among pancreatic Beta cells of euglycaemic rats [19]. C-terminal peptide antibody staining of islets visualised by the $A B C$-peroxidase technique. (A) Low power view of whole islet showing variable glucokinase staining of Beta cells. (B) High power view demonstrating marked differences in glucokinase immunoreactivity. Scale bars represent $10 \mu \mathrm{m}$ 
at higher glucose concentrations. Further investigation is clearly warranted in this area to clarify many unsolved fundamental issues concerning the intra-islet regulation of hormone secretion.

While GLUT 2 and GK may play an important role in insulin secretion as part of the glucose sensor, further characterisation of the distal events that eventually result in exocytosis of the secretory granules is also an important area for further research. Glucose stimulation of islets results in a rapid increase in intracellular calcium levels (see "Ion Channels"). It has been suggested that IP3 produced via activation of protein kinase $C$ may be responsible for this effect by triggering calcium release from the ER. Using a cell-free assay, Okamoto has found that IP3 does not stimulate calcium release from ER microsomes isolated from islets whereas significant IP3stimulated calcium release was observed using brain ER microsomes [21]. Okamoto's group has previously shown that intracellular levels of NAD may regulate insulin secretion, biosynthesis and islet regeneration and that NAD levels are regulated in islets by poly ADP ribose synthetase. The group also described a novel reaction that catalyses the formation of cyclic ADP ribose from NAD. Quite remarkably, when added to islet microsomes, cyclic ADP ribose caused a dose-dependent increase in calcium release. Furthermore, evidence was presented that glucose stimulates the formation of cyclic ADP ribose in islets. These provocative studies raise the interesting possibility that insulin secretion may be regulated by a novel second messenger molecule, in addition to cyclic AMP and other agents such as nitric oxide and cyclic GMP $[22,23]$.

\section{Future directions}

Advances described in this report indicate that our knowledge of the mechanisms that regulate insulin expression and Beta-cell differentiation has reached a level of sophistication sufficient to envisage the construction of artificial Beta cells that could eventually be used in the therapy of diabetes. The important advantages of such cells may well include:

- In contrast to isolated islets obtained from a donor these cells would be available in large quantities.

- Their non-Beta-cell derivation may circumvent autoimmune destruction.

- Genetic engineering of these cells to introduce critical components involved in glucose sensing and stimulussecretion coupling may allow for the development of a cell that secretes insulin under physiological conditions; on the other hand, the repression or down-regulation of potentially harmful products, such as autoantigens or toxic reactions, may improve cellular survival.

- It may eventually be possible to uniquely design these cells for specific targeting in the organism in order to recapitulate regulation of secretion by systemic factors such as gut hormones.

\section{Recommendations}

It is clear from the research overview presented here that much more needs to be learned with regard to the cellular and molecular biology of the Beta cell in order to more fully understand its normal role in physiology and to prevent or circumvent its pathophysiologic deterioration. Among the areas we deem to be most crucial for future research are the following:

- The developmental pathway of the Beta cell through a series of precursor cell stages which undergo successive revisions in gene expression to finally achieve a range of differentiated states that collectively constitute the Betacell phenotype.

- The elucidation of the genes and their protein products that define the Beta-cell phenotype and of those proteins and factors that are required to regulate the expression of Beta-cell-specific genes.

- The mechanisms that regulate the growth, division and regeneration of the Beta cells of the islets of Langerhans so as to enable their controlled expansion or regeneration or both, in vitro and in vivo.

- The cellular mechanisms that regulate proinsulin biosynthesis, processing to insulin and sorting into storage vesicles.

- The mechanisms that regulate insulin secretion involve complex biophysical and biochemical mechanisms of stimulus recognition (i.e. glucose sensing, receptors), stimulus secretion coupling mechanisms (ion channels, G proteins, adenylate and guanylate cyclases, protein kinases and phosphatases) and exocytotic mechanisms (calciummediated membrane fusion processes, etc.) Much more needs to be learned about these processes to begin to attempt to engineer and reconstruct appropriately responding systems and/or to pharmacologically intervene in insulin production and secretion.

- Further analysis of other secretory products of the Beta cells (IAPP, chromogranins, processing enzymes, etc) and of the other islet hormones (glucagon, somatostatin and pancreatic peptide), of their mechanisms of expression in the Beta cell or in other islet-cell populations and of their possible roles in the pathophysiology of diabetes.

Acknowledgements. This report is the result of Work Group 6: Molecular Biology of the Beta Cell at the Third JDF World Con-ference on Diabetes in Monte Carlo, Monaco, 8-10 March, 1992. Chairman: D. F. Steiner. Work group participants: K. Docherty ${ }^{1}$, T. Edlund ${ }^{2}$, P. A. Halban $^{3}$, J. C. Hutton, O. D. Madsen ${ }^{5}$, M. A. Magnuson ${ }^{6}$, C. B. Newgard ${ }^{7}$, H. Okamoto ${ }^{8}$, B. Thorens ${ }^{9}$, L. Villa-Komaroff ${ }^{10}$, Rapporteur: D. E. James. Discussant: D. J. Drucker ${ }^{11}$

${ }^{1}$ Department of Medicine, Queen Elizabeth Hospital, Bimingham, UK:

${ }^{2}$ Department of Microbiology, University of Umea, Umea, Sweden;

${ }^{3}$ Laboratories de Recherche Louis Jeantet, CMU, Geneva, Switzerland;

${ }^{4}$ Department of Clinical Biochemistry, Addenbrook's Hospital, Cambridge, UK; ${ }^{5}$ Hagedom Research Laboratory, Gentofte, Denmark; ${ }^{6}$ Department of Molecular Physiology and Biophysics, Vanderbilt University Medical School, Nashville, Tennessee, USA; 'Department of Biochemistry, Center for Diabetes Research, University of Texas 
Southwestern Medical Center, Dallas, Texas, USA; ${ }^{8}$ Department of Biochemistry, Tohoku University School of Medicine, Miyagi, Japan; ${ }^{9}$ Institute of Pharmacology, Lausanne, Switzerland; ${ }^{10}$ Neurology Research, Children's Hospital, Boston, Massachusetts, USA; ${ }^{11}$ Toronto General Hospital, Toronto, Ontario, Canada.

\section{References}

1. Aronheim A, Ohlsson H, Park CW, Edlund T, Walker M (1991) Distribution and characterisation of helix-loop-helix enhancerbinding proteins from pancreatic Beta cells and lymphocytes. Nucl Acids Res 19: 3893-3899

2. Clark AR, Docherty K (1992) The insulin gene. In: Ashcroft FM, Ashcroft SJ (eds) Insulin, from molecular biology to pathophysiology. Oxford University Press, Oxford (in press)

3. Thor S, Ericson J, Brännström T, Edlund T (1991) The homeodomain LIM protein Isl-1 is expressed in subsets of neurons and endocrine cells in the adult rat. Neuron 7:881-889

4. Lee YC, Asa SL, Drucker DJ (1992) Glucagon gene 5'- flanking sequences direct expression of simian virus 40 large $T$ antigen to the intestine, producing carcinoma of the large bowel in transgenic mice. J Biol Chem 267: 10705-10708

5. Alpert S, Hanahan D, Teitelman G (1988) Hybrid insulin genes reveal a developmental lineage for pancreatic endocrine cells and imply a relationship with neurons. Cell 53: 295-308

6. Madsen OD, Nielsen JH, Michelsen B et al. (1991) Islet amyloid polypeptide and insulin expression are controlled differently in primary and transformed islet cells. Mol Endocrinol 5: 143-148

7. Blume N, Petersen JS, Andersen LC et al. (1992) Immature transformed rat islet Beta cells differentially express $\mathrm{C}$ peptides derived from the genes coding for insulin $I$ and $I I$ as well as a transfected human insulin gene. Mol Endocrinol 6: 299-307

8. Orci L, Vassalli JD, Perrelet A (1988) The insulin factory. Sci Am 259:85-94

9. Halban PA (1991) Structural domains and molecular lifestyles of insulin and its precursors in the pancreatic Beta cell. Diabetologia 34: 767-778

10. Pelham HRB (1990) The retention signal for soluble proteins of the endoplasmic reticulum. Trends Biochem Soc 15: 483-486
11. Lindberg I, Hutton JC (1991) Peptide processing proteinases with selectivity for paired basic residues. In: Fricker LD (ed) Peptide biosynthesis and processing. CRC Press, Boca Raton, pp 41-173

12. Steiner DF (1992) Prohormone convertases revealed at last. Current Biology 1: 375-377

13. Johnson KH, O'Brien TD, Westemark P (1991) Newly identified pancreatic protein islet amyloid polypeptide. Diabetes $40: 310-314$

14. Bell GI, Kayano T, Buse JB et al. (1990) Molecular biology of mammalian glucose transporters. Diabetes Care 13: 198-208

15. Meglasson MD, Matschinsky FM (1986) Pancreatic islet glucose metabolism and regulation of insulin secretion. Diabetes Metab Rev 2: 163-214

16. Unger RH (1991) Diabetic hyperglycaemia: link to impaired glucose transport in pancreatic beta cells. Science 251: 1200-1205

17. Yamada Y, Post SR, Want K, Tager HS, Bell GI, Seino S (1992) Cloning and functional characterisation of a family of human and mouse somatostatin receptors expressed in brain, gastrointestinal tract, and kidney. Proc Natl Acad Sci USA 89: 251-255

18. Hughes SD, Johnson JH, Quaade C, Newgard CB (1992) Engineering of glucose-stimulated insulin secretion and biosynthesis in non-islet cells. Proc Natl Acad Sci USA 89: 688-692

19. Jetton TL, Magnuson MA (1992) Heterogeneous expression of glucokinase among pancreatic beta cells. Proc Natl Acad Sci USA 89: 2619-2623

20. Schuit FC, IntVeld PA, Pipeleers DG (1988) Glucose stimulates proinsulin biosynthesis by a dose-dependent recruitment of pancreatic Beta cells. Proc Natl Acad Sci USA 85: 3865-3869

21. Okamoto H, Takasawa S, Nata K, Yonekura H (1992) Glucoseinduced $\mathrm{Ca}++$ mobilization from islet microsomes is mediated by cyclic ADP-ribose. Diabetes (in press)

22. Laychock SQ, Modica ME, Cavanaugh CT (1991) L-arginine stimulates cyclic guanosine 1', 5'-monophosphate formation in rat islets of Langerhans and RINMSF insulinoma cells: evidence for Larginine: nitric oxide synthase. Endocrinology 129: 3043-3052

23. Schmidt HHHW, Wamer TD, Ishii K, Shung H, Murad F (1992) Insulin secretion from pancreatic $B$ cells caused by L-argininederived nitrogen oxides. Science 255: 721-723 